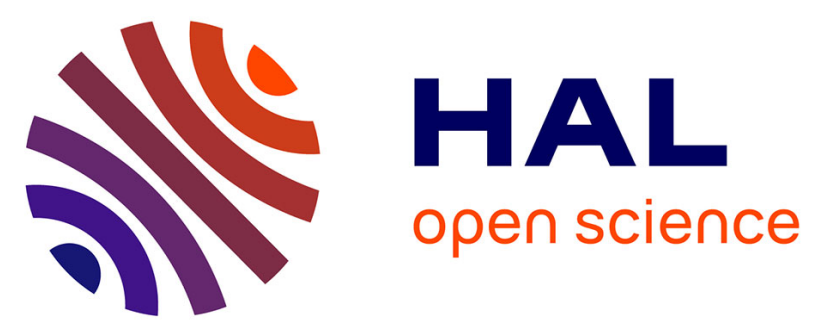

\title{
The Fukushima Daiichi Nuclear Accident: Entering into Resilience Faced with an Extreme Situation
}

Franck Guarnieri

\section{To cite this version:}

Franck Guarnieri. The Fukushima Daiichi Nuclear Accident: Entering into Resilience Faced with an Extreme Situation. Prof. Dr. Joonhong Ahn, Prof. Dr. Franck Guarnieri, Prof. Dr. Kazuo Furuta. Resilience: A New Paradigm of Nuclear Safety. From Accident Mitigation to Resilient Society Facing Extreme Situations, Springer International Publishing - Available under Open Access, pp.1-17, 2017, Print ISBN 978-3-319-58767-7 Online ISBN 978-3-319-58768-4. 10.1007/978-3-319-58768-4_1 . hal01574795

\section{HAL Id: hal-01574795 \\ https:/ /hal-mines-paristech.archives-ouvertes.fr/hal-01574795}

Submitted on 16 Aug 2017

HAL is a multi-disciplinary open access archive for the deposit and dissemination of scientific research documents, whether they are published or not. The documents may come from teaching and research institutions in France or abroad, or from public or private research centers.
L'archive ouverte pluridisciplinaire HAL, est destinée au dépôt et à la diffusion de documents scientifiques de niveau recherche, publiés ou non, émanant des établissements d'enseignement et de recherche français ou étrangers, des laboratoires publics ou privés. 


\title{
The Fukushima Daiichi Nuclear Accident: Entering into Resilience Faced with an Extreme Situation
}

\author{
Franck Guarnieri
}

\begin{abstract}
A transdisciplinary concept, resilience has emerged from monodisciplinary approaches and finds its foundations in various domains such as materials science, ecology, psychology, sociology, ethology, medicine, etc. Although the concept has been a work in progress in the scientific community for several decades, it was only adopted by the safety studies community in the 2000s. The Fukushima Daiichi accident has accelerated its popularity and led to an abundance of theoretical and methodological references, ideas and concepts, processes and approaches that are more-or-less operational.
\end{abstract}

Keywords Fukushima Daiichi - Nuclear accident - Resilience • Entry into resilience $\cdot$ Extreme situation

\section{Introduction}

Unfortunately, dramatic nuclear accidents are a source of information, knowledge and learning for researchers in the safety studies. As their career progresses, members of the community learn more: safety is driven by accidents, catastrophes and disasters of all kinds. While this is obviously a tragedy, it is clear that we do not really know what else to do.

The accident at Three Mile Island on March 28, 1979 taught us that inappropriate actions can lead to core fusion, that serious accidents necessarily involve all stakeholders in civil society, that the defenses of a nuclear installation must be 'deep', and that each accident contains in its genesis 'precursor' scenarios that, if they can be identified, may help to avoid the situation becoming worse [1].

The Chernobyl accident on April 26, 1986, taught us that 'global' public opinion about nuclear safety cannot be ignored [2]. In particular, it showed us that opera-

F. Guarnieri $(\square)$

Centre for Research on Risks and Crises (CRC), MINES ParisTech - PSL Research

University, Sophia Antipolis, France

e-mail: franck.guarnieri@mines-paristech.fr 
tor's cannot act as the sorcerer's apprentice, blithely dispensing with basic safety requirements. It also taught us, a posteriori, that operators must learn from both their own experience and that of others. The concept of 'safety culture' emerged from the Chernobyl accident. It has since been widely popularized and reused to demonstrate that the safety of a facility is the top priority for all operators [3-5].

What have we learned from the Fukushima Daiichi accident?

Two Commissions of Inquiry were established: one at the initiative of Prime Minister Naoto Kan, the other by the Japanese Diet. Both Commissions recognized that the nuclear accident at Fukushima Daiichi was "a man-made disaster" and not simply caused by the earthquake and the giant tsunami that occurred on March 11, 2011 [6, 7]. The Commissions' reports were voluminous, and supplemented by international analyzes [8, 9]. Everything came down to the facts, causes and consequences of the accident. Finally, and as usual, everyone agreed (without actually explicitly saying so) that the accident could be seen as useful, whether in terms of how to regain 'control' of a system that was out of control, or as a way to learn from each other's mistakes, or as example of the intertwined decisions that were taken at multiple levels (local, hierarchical, organizational, inter-organizational, political, international, etc.). In other words, as a way to say that all of this could have been avoided if only...

All of these Commissions produced a long list of recommendations that are both sensible and helpful in improving safety. However, fundamentally they offer nothing new - all are consistent with a 'normative' vision of nuclear safety. Although it cannot be disputed that they are an invaluable source of knowledge and progress, their very nature creates a form of myopia. This myopia is so pronounced that it eventually produces expert and techno-centered analyses that only take into consideration standards, laws, regulations and procedures. It excludes all forms of humanity from a human activity, while accidents are clearly a departure from the logical course of events.

Does this mean that the accident at Fukushima Daiichi will have taught us nothing? Obviously not! Rather, it places the concept of resilience, which has become particularly fashionable in many disciplines, at center stage in nuclear safety. The concept was integrated into safety sciences in the early 2000s. It has taken pride of place in the context of the Fukushima Daiichi accident. Entering into resilience assumes the system has survived, if not it has perished!

This chapter is organized into five sections. It identifies and describes the determinants of the entry into resilience in a socio-technical system that is the victim of an unprecedented accident which, without an adequate response, will obliterate the system itself. The first section revisits the notion of the accident, looking at it in terms of the 'extreme situation'. The second introduces the concepts of resilience and entry into resilience. The third and the fourth sections respectively discuss the link between the entry into resilience and notions of time and space. The last section discusses the human and organizational determinants of an organization's entry into resilience. The Fukushima Daiichi accident, and in particular the decisions taken by the engineering teams on the site between 11 and 15 March 2011 serve as a case study. 


\section{From Nuclear Accident to Extreme Situation}

Fukushima Daiichi is a Japanese nuclear power plant, which on March 11, 2011 suffered, like the rest of Eastern Japan, the effects of a terrible earthquake followed by a devastating tsunami. Before becoming a nuclear power plant, the site was a training camp for the Japanese kamikaze during the Second World War [10]. At the time of the accident, the Director of the plant was Masao Yoshida. The Daiichi plant is a neighbor to the Dai Ini plant, where Naohiro Masuda was the Director, and whose handling of the crisis was presented as a model of good management [11]. Like Masuda, Masao Yoshida knew perfectly well how to handle the situation (which we will later term 'extreme') he and his men were faced with, despite the fact that the damage and losses were far more extensive at Daiichi than Daini. Nevertheless his actions came under severe criticism. But that is the subject of another story [12].

The management failures that occurred in the handling of the crisis at Fukushima Daiichi are not the first of their kind: the accident at Three Mile Island, not to mention Chernobyl had already highlighted the inability of crisis management procedures to cope with 'unthinkable' situations. From this, it seems almost reasonable to conclude that few real lessons have been learned [13].

There appears to be a kind of illusion of safety, a fact underlined by the pertinent observation of a TEPCO manager who explained the accident in terms of overconfidence, lack of imagination and various biases [14]. Despite all efforts to overcome them, these three challenges lead to accidents that Perrow [15] describes as "normal" or "systemic", due to complex interactions and tight coupling within the system. However, the phenomenon described by Perrow is not new. The historian Fressoz [16] describes its origins in the nineteenth century with the development of the railway system; catastrophes have proven to be inherent in systems where "nobody is able to anticipate and ward off the effects" [17].

While it is recognized that failures are inevitable, contemporary approaches to 'beyond-design-basis' accidents in nuclear safety [18] must address ongoing challenges. In the aftermath of extensive damage, actors must adopt innovative, improvised solutions to return to a safe situation. Similarly, they must draw upon resources that are not part of the usual frameworks and patterns for problem solving [8]. It is clear that this postulate is far from being accepted. The numerous investigations into the accident repeatedly reaffirmed the benefits of the concept of defense in depth [19], despite its inability to effectively evaluate events that lead to the emergence of new sources of vulnerability [9].

The situation at Fukushima Daiichi goes beyond coping with an unthinkable disaster, and concerns "a state of emergency which seems to have no end", or even "a slow, diluted catastrophe, an ongoing catastrophe" [20]. This has consequences for how we handle the phenomenon. The Fukushima accident is not limited to the 
period from 11 to 15 March 2011; the site is subject to ongoing natural threats (from another earthquake or tsunami) and the facilities remain severely damaged.

When question of when the accident will end raises the issue of uncertainty in a post-accident context where many parameters cannot optimized. One of the lessons learned from the Fukushima Daiichi crisis is that many countries now consider the post-catastrophe phase of preparations for a "return to normal" to be equally as strategic as crisis management preparations [9]. Confirmation comes from the Director of the Fukushima Daiichi plant, Masao Yoshida. Yoshida testified that the operator, TEPCO, had not provided any emergency measures in the case of a power failure, which meant that there was no way to cool the reactors [21]. Even today, certain events remain unexplained and there are clear cases of ambiguity. ${ }^{1}$

Therefore, rather than the concept of the accident, a more useful concept appears to be that of the 'extreme situation'. Gilbert [23] argues that "some situations have become real black boxes" due to a blatant lack of investigation and analysis. Safety approaches are limited to compliance with risk analysis principles and standards, and therefore tend to underestimate extreme phenomenon that cannot be planned for.

A situation is called 'extreme' when conditions are radically different from those of so-called 'normal' life and are unusually intense, becoming excessive, or even unbearable [24]. Dealing with the extreme situation pushes people to their limits; to the edge of the abyss [10]. The individual, group, organization, company, or more simply, the system is faced with extreme violence, a radical shake-up of life as they know it.

The extreme situation leads to the destruction of identity, the loss of benchmarks and frames of reference. The explanation is simple; identity is shaped or manufactured by external relationships (specifically, compliance) with current social norms, adherence to common and therefore shared values, responses to social expectations, and dependency or even subordination between actors in the system. From the moment the (existing) value system is shattered, a change occurs - and a new system appears.

The concept of the extreme situation therefore places the individual and the organization that must face the unthinkable at the heart of the analysis [24]. The unthinkable takes the form of three 'entities' that become, through the forces of nature and human weakness, uncontrollable and 'unleashed'. Following a period of 'devastation' and predictions of 'certain death' [25], the actors involved in the Fukushima Daiichi accident began a phase of 'coping', which enabled them to mobilize multiple resources in order to survive in the short term [26]. They then began a return to an acceptable situation, despite extensive damage, widespread pollution and the hazards that endangered, and continue to endanger the site.

\footnotetext{
${ }^{1}$ For example, the causes of the malfunction of the core of the reactor cooling system (Reactor Core Isolation Cooling) of Unit 2 remain unclear [22].
} 


\section{Entry into Resilience: A Way to Cope with the Extreme Situation}

The concept of resilience is only relevant following damage, loss, an accident, trauma, etc. Pre-event is the domain of prevention, prudence, or even precaution. To be able to discuss resilience, you first have to survive. Even this is not enough, as, while it can be a sustainable situation, survival is fragile. The concept has positive connotations. Resilience is an asset, it represents progress; being or becoming resilient does not mean returning to the nominal, pre-shock state. This is anyway impossible because the system, whatever form it takes, remembers the event (albeit for a limited period of time).

Although the concept is in fashion, there is no universal definition that can be applied to all domains. That said, the English term 'resilience', itself derived from the Latin verb resilire (to bounce), is made up of re (again) and salire (rise), which implies a retroactive effect [27]. While in the 1970s the term was associated with the ability to absorb and overcome the effects of significant, unexpected and brutal disruption to ecological systems [28], hybrid definitions have since emerged in many disciplines including geography [29], psychology [25], sociology [30], organizational sciences [31], ergo-psychology [32], etc. Within this smorgasbord of definitions, two fundamental ideas prevail: community, ${ }^{2}$ and the process. ${ }^{3}$

In the absence of a consensus, resilience can be defined as the capacity of a system to absorb disturbances and reorganize itself during ongoing changes [33]. It is probably more relevant, especially in the case of an accident as serious as that Fukushima Daiichi, to place less emphasis on states of equilibrium as "frontiers as a function of the domain of attraction" because paradoxically, highly fluctuating instability can also foster entry into resilience [28]. In practice, a system can be very resilient, yet fluctuate significantly and therefore be fairly unstable. This approach seems more relevant in the case of Fukushima Daiichi where, given the enormity of the shock, it was more important to preserve relationships in the socio-technical system than to return to the previous equilibrium as quickly as possible, which in fact proved to be an unrealistic expectation [34].

Contemporary views of nuclear safety see the concept of resilience as a post-crisis process, part of a community dynamic that stresses organizational adaptability [35]. It has a predictive dimension that helps the organization to overcome adversity and get back on track [27]. However, this predictive dimension, and a fortiori entry into resilience, must not neglect the role of probability, uncertainty [13] or even a "surprise" dimension in the success (or failure) of its implementation [36]. Here again, there is a reference to a conscious capacity to "navigate" and "negotiate" [37] in order to cope with an extreme situation.

\footnotetext{
${ }^{2}$ In ecology, a community is a group of organisms belonging to populations of different species making up a network of relationships.

${ }^{3} \mathrm{~A}$ process is a system of activities that uses resources to transform inputs into outputs.
} 
The term 'entry' expresses "movement from one place to another" [38]: therefore there is a 'transition' that must be taken into account. The concept is also linked to the question of "the place through which we enter" [38], and thus the direction of the transition. Time also plays a part, as the 'entry into' begins with the exercise of "a practice" [38] that implies a "change of state" [39].

The change of state is explained by unforeseen sequences of complex interactions that could not be predicted [15]. Entry into resilience must therefore be perceived as an 'exploratory zone' in which it is necessary to have a better understanding of the interactions in order to improve how they are managed. From this point of view, a learning phase is necessary. It is difficult to rationalize this exploration phase either upstream or during the process as it resembles "cognitive DIY that does not belong to the scientific world (which does not mean that it cannot be effective)" [40]. In this case, rationality could be described as procedural, with "simple but strict rules, which certainly does not completely eliminate risk, but reduces it to a level below that resulting from substantive rationality" [41].

Entry into resilience therefore translates into the creation of a new system [33] when ecological, economic or social conditions make the initial system untenable. In this case, the variables and scope that define the system must be modified. Nevertheless, the potential for the loss of a certain degree of resilience is inevitable, in the context of the dynamic interactions found in adaptive complex systems. Such multiple interactions were thrown into sharp relief during the Fukushima Daiichi crisis [8] and complicated the implementation of appropriate responses.

It is important to note that older definitions include an element of 'privilege', linked to the capacity to 'enter into'. The chambers of the king of France could only be visited at specific times [42]. By extension it could be argued that this 'entry into' also embodies a situation where the parties involved must demonstrate in advance their capacity to access the privilege: in other words, education, training, experience, professionalism, etc. The curriculum vitae of Masao Yoshida [12] is illuminating in this sense as it demonstrates his competence and expertise, making him the right person in the right place.

Finally, the notion of 'entry into' finds support from the biological metaphor of the membrane, "a generic organ that links the interior with the exterior, the past and the future, using the dual mode of qualification/interpretation of the future through the past, and the integration of the future using the encoding of the past" [43]. This metaphor enables the introduction of the relationship between time and space.

Prigogine [44] places the question of time "at the crossroads of problems of existence and knowledge". In this sense, his argument is similar to that of Heidegger [45] for whom the question "of the meaning of being" cannot be examined without an interpretation of time as the possible horizon for any understanding. If it is accepted that resilience is a process, and that the entry into resilience is a moment (an instant or a short duration...), then it cannot be argued any other way than that time is one of the determinants that shapes its nature. If it is accepted that time is "at the crossroads", the question of space necessarily arises. Time and space are therefore the most important, fundamental and essential determinants to be examined. 


\section{Entry into Resilience and Time}

Time is "huge and complex" [46]. Classically, it is approached from three angles: chronology (a sequence of events that follow one another), simultaneity (events that occur at the same time) and duration (a measurable period). Typically, a linear and cumulative concept is contrasted with one that takes into account multiple, discontinuous temporalities. For example, [47] summarizes it as, "rather than simulate a linear story that is 'in progress', priority should be given to these flashbacks, these evil blows, these lightweight catastrophes that perturb an empire much better than major upheavals. Give priority to non-linearity, reversibility, everything that relates, not to an unfolding or an evolution, but a winding game, a reversion in time. Anastrophe versus catastrophe. (...). Everything happens in loops, in tropes, in reversals of direction". The challenge is to know how to manage these various feedback loops, "the analyzed object's victorious ruse" [48] especially within a system in crisis.

The concept of crisis can be defined as a runaway phenomenon, the translation of an acceleration, or a loss of control. Entry into resilience aims to halt this phenomenon using a more powerful natural or artificial mechanism, which will initially slow it down and then stop it [49]. More specifically, in the case of an extreme situation the focus is less on a search for the causes of this runaway phenomenon, than to understand the "delirium of forms and appearances", this "endless cycle of metamorphoses" where everything "explodes into connections". It could be said that entry into resilience helps to "slow down, stop at certain points this total correlation of events" [48]. It is therefore essential to have feedback loops [50] that act retroactively on the source. From this perspective, entry into resiliency can be seen as an attempt to create a return to the origin of the crisis so that the system can be brought back under control and become more fluid.

The challenge is therefore to find the right tempo ${ }^{4}$ that allows the technical objects $^{5}[51]$ in the system sufficient time to independently enter into resilience. This tempo is punctuated by 'phases' that have a role to play, but "by phase, we do not mean a moment in time that is replaced by another". It is more accurate to say that "in a system there are phases when reciprocal tensions are in equilibrium; the current system composed of all phases taken together is that the full reality, not each phase taken by itself, a phase is only a phase when compared to another. (...). Finally, the existence of a plurality of phases defines a neutral, balanced center, which provides the conditions for the existence of the diphase" [52]. An overall harmony with respect to time management must therefore emerge. In the time management context, it is in the relaxation phases where minor or major factors are

\footnotetext{
${ }^{4} \mathrm{Here}$, the concept of tempo is not limited to the speed of execution but the pace at which a set of actions unfolds.

5"The configuration of technical objects specifies a certain division between the physical and social world, assigns roles to certain types of actors - human and nonhuman - and thereby excludes others, permits certain modes of relationship between these actors, etc.” (p. 49).
} 
synchronized. In practice, there is period of relaxation that is proper to the technical object, which should be respected.

This issue of tempo is generally adapted in improvisation phases. Weick [53], drawing on the work of Berliner [54] and his study Thinking in Jazz: The Infinite Art of Improvisation, argues that at the organizational level improvisation involves reworking pre-composed material and considering it in relation to unanticipated concepts that are designed, formed and transformed under specific performance conditions. Jazz is not spontaneous, intuitive music but flows from the experience of 'musician/actors' and the disciplined application of a vast musical knowledge. This analysis can be expressed in the context of the improvisation of actors involved an extreme situation, who must rely on a repertoire of training resources, experience and a shared vision [55].

A dual temporality must be also managed [56]. The first requires a short-term response that should not limit the adaptability of the system in the long term. The second involves establishing a balance between the need for resources to be mobilized in the very short term and other resources that need to be saved for the long term [32].

Finally, time cannot be discussed without the notion of duration [57]. Bergson opposes the idea of the duration of consciousness and scientific time (defined in terms of measurable periods). Instead, time is the measurement of repetition in space. It is thus a way to reduce an evolutionary phenomenon to spatial coordinates. "Pure duration" is unconstrained thought in the timeline of the same evolutionary phenomenon. It implies something that ensures continuity between successive states. According to Bergson this is the consciousness of the observer, which he calls "intuition", because it is not perceived through a projection of the evolution of the system described in spatial terms, but by a thought that is inseparable from its object.

\section{Entry into Resilience and Space}

The idea of space, inseparable from that of time, is classically considered in the broad and Cartesian sense that defines it as a "scope, a medium in which the observed phenomena occur or abstractions are the object of study" [58].

The viability of a technical object is intimately linked to its "associated milieu" [59] or even a "mixed milieu, both technical and geographical" [52]. The severity of this local challenge was severely underestimated in the spatial management of the Fukushima Daiichi accident. It has even been argued that in general, the remote interaction between the various organizations that should have been responsible made it "pitiful" [60]. An example is the relationship between Yoshida (who considered senior managers to be disconnected from reality) and TEPCO headquarters-when his request for 4,000 tons of water was understood by management as 4000 tons of drinking water [61]. Frustrated, the plant's director gave greater weight to the local situation, to the extent that he decided to ignore certain orders 
from headquarters. The notable example is when he decided to continue (after checking with his subordinate) operations to inject seawater to cool Reactor 2 [62]. However, most of the local initiatives taken by Masao Yoshida only proved relevant in a posteriori debates and controversies. An example is his decision to move skilled workers from Reactor 1 to a more secure building further away for their own protection. Paradoxically, some of these workers criticized the decision, which they perceived as demeaning [61].

It can be difficult to identify the perimeter of the local zone, and a fortiori that of the zone for entry into resilience. Nevertheless, it seems very likely that it should be as close as possible to where the accident took place. Thus, for example, the support of the United States (which is also indicative of an ambiguous state of dependency) was not always very useful. An example is that American authorities initiated the assembly of four water pumping systems for each of the plant's ponds. However, on March 18, 2011, when the parts had already been assembled in Australia and were waiting to be transferred by plane to Japan, the Americans learned that the Tokyo Fire Department had been able to build a pump that was similar to the equipment already used at the site [63]. This failure can be explained by the argument that the nuclear industry is "essentially a centralized energy" [59]. A crisis of the magnitude of Fukushima Daiichi requires a level of decentralization that is not found in its culture. The extent of this centralization is most clearly seen at the time when the crisis began (between Friday, March 11 and March 12 at 10 am), when TEPCO's most senior managers, Tsunehisa Katsumata (Chairman) and Masataka Shimizu (President), were respectively on business trips to China and Nara (an historical Japanese city) [64]. Their absence meant that TEPCO was unable to make quick decisions. This trend towards centralization often results from an exaggerated fear of panic, which must be avoided at all costs, but that actually occurs very rarely [65].

The importance of the local management of the crisis at Fukushima Daiichi has been highlighted in numerous analyzes and reports [9]. Following the catastrophe, some countries re-evaluated the size and nature of their emergency evacuation zone, according to the country and the geographical context of its nuclear plants. Developing cooperation with neighboring countries in the case of emergencies has become a new priority, in particular joint maneuvers and exercises.

Local solutions must therefore be established to facilitate and accelerate entry into resilience. These solutions can, for example, be based on the pertinent criterion of "concentration" [65] (of energy, populations and decision-making powers).

In this context, the concept of Community Building Recovery Corporations (CBRC) [66] seems to be relevant. It is based on three levers: change, community and leadership. Economic reconstruction (i.e. post-accident) is established on a local level with the support of citizens in a combined public-private approach. The resources that are mobilized are primarily local, guided by the principle of a high level of creativity in their implementation. The Fukushima accident demonstrates the usefulness of the CBRC concept, as the main challenges concerned not only electricity and information, but also the more basic problems of a lack of food, 
access to toilets and additional emergency rescue teams [8], which could have been better solved locally.

However, in a nuclear crisis decentralized management is not sufficient for success. The Chernobyl catastrophe was primarily managed in a decentralized way and clearly ended in failure. "The accident analyzes give the impression that the workers at the bottom of the ladder ran the system in their own way, trying to cope with the facility, to gain time and to fix things, incredible acrobatics" [40]. Moreover, the arrival of engineers from Moscow proved ineffective as they were highly specialized in electro-mechanics and not the operation of a nuclear reactor (and knew even less about nuclear safety). Consequently, this failure of decentralized management at Chernobyl led operators and regulatory authorities to favor a technical approach that limited the room for maneuver of operational staff. However, the Fukushima Daiichi accident poses a challenge to this approach.

Beyond the issue of decentralization, entry into resilience also raises new questions on the theoretical level, notably regarding the path of this "entry" and the definition of a space dedicated to it. This requires a change in the formalization of objectives, and consequently the trajectory of the socio-technical system [32]. The approach differs from ecological definitions of the concept of resilience that tend to focus on the capacity (or not) of a community or group to confront and overcome adversity, and therefore to enter into resilience. A perspective framed in terms of trajectory places the emphasis on essential and vital functions that must continue after a crisis, something that seems more relevant in the framework of successful entry into resilience. Moreover, this trajectory must move in the direction of a "basin of attraction" in which systems tend to reach equilibrium [33]. This equilibrium must play the role of attractor in guiding the trajectory of entry into resilience and result in "stability landscapes" that are defined by clear boundaries. Stabilization can be disrupted [67] by both external elements (for example, conflicting orders from headquarters or the government) and internal elements (for example, lack of data, or undue, and ultimately mistaken reliance on information provided by a sensor).

In practice, the path is more like a rotary (roundabout) than it is linear [68], as each actor can enter or leave at any point to enter into resilience, while a kind of 'insider/outsider' dynamic can be implemented in neighboring communities. The trajectory can also be oriented in such a way as to fill the structural voids at the borders of organizational units [69], which become strategic hubs. A risk related to the orientation of this trajectory is 'destinationism' [41], which consists in having an excessive focus on the target to be met. However, in the case of an extreme situation that is highly unstable, it is sometimes necessary to adjust the target. Finally, establishing a clear and limited objective presupposes that complex, uncontrolled interactions can be understood.

The capacity to implement a "pivot strategy" is essential. This strategy can increase "resilience capital" [70] and as a result facilitate entry into resilience. To extend and improve resilience, organizations must also encourage heretical questions at operational levels [71]. 
We now address the question of the formalization of a space dedicated to resilience and entry into it.

"Scoping" [72] is designed to clarify the boundaries of the organization in relation to a given area. In the case of a nuclear accident, although the management of the organization is limited to certain target areas, it is important to maintain a holistic view of the crisis. The Cynefin model [73], for example, distinguishes four categories of context that can help in understanding an extreme situation:

- Chaotic context: there does not seem to be any link between causes and their effects. The available concepts are useless. An open-minded approach, focused on investigation and exploration is essential before taking decisive action and analyzing its effects. Under time pressure, action must be both rapid and carefully considered.

- Complex context: there are multiple interactions between causes and their consequences but it is impossible to precisely model or predict the impact of an initiative on the system. In this context, an insightful and flexible exploratory analysis should be carried out.

- Knowable context: the relation between cause and effect can be assessed but there is not enough data to be able to evaluate the situation.

- Known context: the relation between cause and effect is predetermined.

From the perspective of trajectory, entry into resilience consists in moving from a chaotic context to a complex context. The situation at Fukushima Daiichi will only be fully brought under control when a known context is achieved. Current conditions at the site are still complex and fragile: the return to a chaotic context cannot be excluded if, for example, another major earthquake occurs. This shows that entry into resilience does not guarantee a sustainable trajectory in the long term.

It is important to note that the transition from one context to another or, more generally, entry into resilience implies a period of adaptive research. During this period, actors establish trade-offs as a function of boundaries between acceptable and non-acceptable performance [74]. If it proves impossible to avoid crossing a boundary, an error or accident may occur. There is therefore a 'dead point', beyond which entry into resilience reaches a point of no return and will fail. This point represents a threshold where any system that "crosses this fine line of reversibility, contradiction, questioning" will "enter, living, into its own frenzied contradiction..." [48] and ultimately perish.

Therefore, entry into resilience implies that the actors know how to delimit the intervention space and can establish boundaries beyond which the situation is irreversible. In an emergency, it is likely that in most cases the concept of entry into resilience and its relation to boundaries is metaphoric, and cannot be quantified in terms of risk [32]. Nevertheless, the understanding of risk must be communicated internally to actors at all levels of managerial responsibility [75].

We should not overestimate aspects of local culture in the management of resilience and thus the establishment of a resilient space. It is more important to observe the influence that certain forms of culture have on an organization. 
For example, entry into resilience involves a shift from a pathological and bureaucratic culture (notably embodied in the relationship between TEPCO and Japanese political powers), to a generative culture that enables the organization in crisis to activate resilience in the best possible conditions [76]. It should be noted that this argument refers more to general boundaries than a definition sensu stricto of measurable organizational boundaries.

In summary, the questions of the path for entry into resilience and the definition of its space are acute. Going beyond the models of Snowden [73] and Westrum [76], a more accurate analysis of the Fukushima crisis will help to formalize and theorize trajectories and spaces necessary for entry into resilience by paying greater attention to the details of how operations unfolded.

\section{Human and Organizational Factors of Entry into Resilience in an Organization}

Many models $[15,18,32,65,69,74,75]$ describe the response of organizations to disruption at all orders of magnitude. Most rely on a call for strategic and operational, or even financial resources. Some also examine the safety management system and attempt to determine if a system is resilient or not. Although these contributions are clearly important, in our view they overlook the dynamic character of the resilience process. In fact, there are very few models that describe the mechanisms and determinants through which resilience emerges in response to a traumatic event.

Two key processes in the mobilization of resilience can be cited: the reconfiguration of resources and their mobilization [77].

Resilience may emerge when "resources are sufficiently robust (...) or rapid" [68] to be able to slow down and counteract the negative effects of a severely degraded environment. A key concept that quickly emerges is the "conservation of resources". According to the theory of the same name (Conservation of Resources, COR) [78], stress emerges when resources are limited, lost or when individuals cannot replenish them despite significant effort [68]. COR theory argues that actors must invest in resources to guard against the loss of other resources. In some ways, entry into resilience makes sense in this context: it makes it possible to anticipate the loss of valuable resources and thus curb fears of a runaway situation that could lead to the loss of the system itself.

In terms of resource mobilization, the work of Powley [79] on the concept of "resilience activation" is particularly interesting. Entry into resilience is established as a result of three mechanisms that follow each other in chronological order. Resilience is activated during a critical period. Its smooth operation implies first and foremost "liminal suspension", which in turn activates "compassionate witnessing" and then "relational redundancy". It is in these conditions that a resilient organization is created. 
Liminal suspension activates the resilience of the organization in two ways. First, it provides a temporary holding space in which its members have time to readjust on a psychological, emotional and relational level. Members of the organization can help and support each other without work constraints in a "holding space for pain". Second, the crisis can shift the positions of actors, leading to the creation of new relational structures and the strengthening of existing relationships. The social positions of some members of the organization may shift as new relations that create solidarity are formed. Liminal suspension activates resilience by undoing social and organizational structures for a period of time in which actors lose their 'status' and new, deep bonds are formed that challenge previous relationships. It also creates time and space for relational structures to shift, thereby "loosening control" to reduce "defensive perceptions" between members of the organization [80]. However, liminal suspension does not make the distinction between roles obsolete.

"Compassionate witnessing" implies empathy for others. This phase follows on from the new relational structures that emerged in the previous phase. Compassionate witnessing can be activated in two ways within an organization. The first is to demonstrate mindfulness in relationships with others. This dimension is not only limited to cognitive aspects, but also implies identifying the emotions of others, leading to an appropriate response to their emotional, physical and social needs. The second is sharing and connecting. In particular, sharing experiences helps the organization's members to restore order and bounce back. Compassionate witnessing activates resilience by adapting the organization to the response of its members. In this case it does not coordinate behavior, but rather emphasizes the importance of emotions and thoughts in the interpersonal relations that enable actors to heal from the trauma.

"Relational redundancy" refers to interpersonal connections that overlap and intersect, and extend beyond the boundaries of the social reference group. There are two aspects to relational redundancy. First, there is an informational connection that relates to the ability of members of the organization to share critical information about safety. Secondly, overlapping social links provide a holistic and panoramic view of the entire set of interactions within the organization. Relational redundancy therefore activates resilience through intersecting interactions that ensure the persistence of relationships within organizational systems. From this perspective, the informational field widens when actors share critical information within the system or with their immediate social reference group. Counter-intuitively, resilience is activated not through principles of organizational efficiencies, but through principles of redundancy and excess relational capacity. From this perspective, each actor in the system plays a strategic role in finding and transmitting critical information to other members of the organization. This abundance of connections favors the emergence of a new kind of network that creates multiple opportunities for members to share information, express emotions and therefore enable organizational resilience. 


\section{Conclusion}

Characterizing the determinants of entry into resilience is a major challenge. It is clear that very little is known about it. Some disciplines (for example, psychology and ecology) have made more progress than others. These disciplines have the advantage that research is based on a clinical approach, and they are able to examine extremely well-documented cases. However, the challenge that remains is to generalize the examination of a specific and particular situation. In the fields of management science and human resources, the work of Powley [79] is particularly interesting. The author proposes a data analysis methodology and a model for determinants that are very relevant to our understanding of the mechanism for entry into resilience. The field of safety sciences has made its own contribution. The work of Hollnagel [32] provides a definition of the concept of resilience and its application to the measurement of the performance of a safety management system on the one hand, and, on the other hand, four meta-categories (respond, monitor, anticipate, and learn) that offer other ways to investigate the issues in more depth. While the number of conceptual models that are aimed at standardization is legion, the fact remains that little is known about the actual teams, groups, and organizations that must face the unthinkable. This lack of knowledge favors an approach in the field that tries to immerse itself as deeply as possible into the mechanisms and processes that come into play when facing an extreme situation. The Fukushima Daiichi accident offers an extraordinary field for researchers by virtue of the profusion of data sources and the information that has already been made public and which will eventually be made public in the future. One example is the hearing attended by the plant's Director [12]. His very personal testimony helps us to understand what motivated the responses of engineering teams who were faced with an unprecedented situation and offers an initial starting point for thinking about the concept of engineering in extreme situations [81].

\section{References}

1. United States, President's Commission on the Accident at Three Mile Island, Kemeny, J.G., The Need for Change: The Legacy of TMI (United States Government Printing Office, 1979)

2. P. Tanguy, Les leçons de Tchernobyl. Conclusions of the SFEN-SNS colloquia held at Paris 15-17 April 1991. Revue Générale Nucléaire, (2), 157-157 (1991)

3. IAEA, International Atomic Energy Agency, Summary Report on the Post-Accident Review Meeting on the Chernobyl Accident, International Nuclear Safety Advisory Group (Safety Series 75-INSAG-1), Vienna (1986)

4. IAEA, International Atomic Energy Agency, Safety Culture, International Nuclear Safety Advisory Group (Safety Series 75-INSAG-4), Vienna (1991)

5. IAEA, International Atomic Energy Agency, Examples of Safety Culture Practices (Safety report series No 1), Vienna (1997) 
6. The National Diet of Japan Fukushima Nuclear Accident Independent Investigation Commission, The Fukushima Daiichi Nuclear Power Situation Disaster: Investigating the Myth and Reality (Ed. Routeldge/Earthscan, New York, 2014)

7. ICANPS, Investigation Committee on the Accident at Fukushima Nuclear Power Stations of Tokyo Electric Power Company, Executive Summary of the Final Report (2012)

8. The National Academy of Science, Lessons Learned from the Fukushima Nuclear Accident for Improving Safety of U.S. Nuclear Plants (The National Academies Press, Washington, 2014)

9. The Fukushima Daiichi Nuclear Power Plant Accident: OECD/NEA Nuclear Safety Response and Lessons Learnt. OECD 2013. NEA No. 7161

10. R. Kadota, On the Brink: The Inside Story of Fukushima Daiichi (Kurodahan Press, 2014)

11. R. Gulati, C. Casto, C Krontiris, How the other Fukushima plant survived. Harward Bus. Rev.

12. F. Guarnieri, S. Travadel, C. Martin, A. Portelli, A. Afrouss, L'accident de Fukushima Daiichi; Le récit du directeur de la centrale, vol. 1 (Presses des Mines, Paris, L'anéantissement, 2015)

13. E.M. Geist, What Three Mile Island, Chernobyl, and Fukushima can teach about the next one. Bull. Atom. Sci. (2014)

14. K. Benedict, The myth of absolute safety. Bull. Atom. Sci. (2014)

15. C. Perrow, Normal Accidents, with a New 'Afterword' (Princeton University Press, Princeton, 1999)

16. J.-B. Fressoz, L'apocalypse joyeuse, une histoire du risque technologique. Seuil (2012)

17. F. Tourneux, Encyclopédie des chemins de fer et des machines à vapeur (Renouard, Paris, 1844)

18. M.A.B. Alvarenga, P.F. e Melo, Including severe accidents in the design basis of nuclear power plants: An organizational factors perspective after the Fukushima accident. Ann. Nucl. Energy 79, 68-77 (2015)

19. International Nuclear Safety Advisory Group, Defence in Depth in Nuclear Safety (IAEA, Vienna, INSAG-10, 1996)

20. M. Ferrier, Fukushima, récit d'un désastre. Folio Gallimard (2012)

21. The Japan Times, Heavy control consol falls back into Fukushima fuel pool: Tepco, August 292014

22. S. Mizokami, Y. Kumagi, Event sequence of the Fukushima Daiichi accident, in Reflections on the Fukushima Daiichi nuclear accident (Springer, 2014), pp. 1-17

23. C. Gilbert, Quels risques pour la recherche en sciences humaines et sociales. Dans Bourg D., Joly P-B., Kaufmann, A. (Dir.), Colloque de Cerisy, Du risque à la menace. Penser la catastrophe. PUF (2013)

24. G.N. Fischer, Le ressort invisible. Paris, Seuil, republished by Dunod (1994)

25. G.E. Richardson, The metatheory of resilience and resiliency. J. Clin. Psychol. (2002)

26. L. Pearlin, C. Schooler, The structure of coping. J. Health Soc. Behav. (1978)

27. A CARRI Report, Definitions of community resilience: an analysis (2013)

28. C.S. Holling, Resilience and stability of ecological systems. Annu. Rev. Ecol. Syst. 4, 1-23 (1973)

29. N. Adger, Social and ecological resilience: are they related? Prog. Hum. Geogr. 24(3), 347-364 (2000)

30. S. Saint-Arnaud, P. Bernard, Convergence or resilience? A hierarchical cluster analysis of the welfare regimes in advanced countries. Curr. Sociol. 51(5), 499-527 (2003)

31. P. Reinmoeller, N. van Baardwijk, The link between diversity and resilience. MIT Sloan Manage. Rev. (2005)

32. E. Hollnagel, D. Woods, N. Leveson (eds.), Resilience Engineering-Concepts and Precepts. Ashgate, (2006)

33. B. Walker, C.S. Holling, S.R. Carpenter, A. Kinzig, Resilience. Adaptability and Transformability in Social-Ecological Systems, Ecology and Society 9(2), 5 (2004)

34. M. Bunn, O. Heinonen, Preventing the Next Fukushima. Science, 333 (2011) 
35. A. Boin, A. McConnell, Preparing for critical infrastructure breakdowns: the limits of crisis management and the need for resilience. J. Conting. Crisis Manage. 15(1) (2007)

36. T. Aven, On some recent definitions and analysis frameworks for risks, vulnerability, and resilience. Risk Anal. 31(4) (2011)

37. M. Ungar, Resilience across cultures. British Journal of Social Work 38(2), 218-235 (2008)

38. A. Rey, Le Petit Robert (2012)

39. C.S. Holling, Engineering resilience versus ecological resilience, in Engineering within Ecological Constraints (1996), pp. 31-44

40. C. Morel, Les décisions absurdes. Gallimard Folio Essais (2002)

41. C. Morel, Les décisions absurdes II (Comment les éviter, NRF Gallimard, 2012)

42. P. Dibie, Ethnologie de la chambre à coucher (Vol. 4). Editions Métailié (1987)

43. J.H. Barthélémy, Cahiers Simondon, N4, L’Harmattan, 15 April 2012, (2012), pp. 23-24

44. I. Prigogine, Laws of nature, probability and time symmetry breaking. Physica A 263(1), 528-539 (1999)

45. M. Heidegger, Being and Time: A Translation of Sein und Zeit (SUNY Press, 1996)

46. M. Paty, Sur l'histoire du problème du temps. Le temps physique et les phénomènes. In Le temps et sa flèche. Editions Frontières (1994)

47. J. Baudrillard, L'illusion de la fin ou la grève des événements. Galilée (1992)

48. J. Baudrillard, Les Stratégies fatales. Grasset Livre de Poche (1983)

49. M. Foucault, Sécurité, Territoire, Population, Cours au Collège de France, 1977-1978 (Gallimard, Seuil, 2004)

50. J. Ellul, Le bluff technologique. Pluriel (2012)

51. M. Akrich, How can technical objects be described, in International Workshop on the Integration of Social and Historical Studies of Technology, Enschede, vol. 3, no. 5 (1987)

52. J.-Y. Chateau, Le vocabulaire de Simondon. Ellipses (2008)

53. K. Weick, Improvisation as a mindset for organizational analysis. Org. Sci. 9(5) (1998)

54. P. Berliner, Thinking in Jazz: The infinite art of improvisation (University of Chicago Press, Chicago, 1994)

55. T. Wachtendorf, K. Kendra, Improvising Disaster in the City of Jazz: Organizational Response to Hurricane Katrina (Social Sciences Research Council, 2006)

56. K. Tierney, The Social Roots of Risk, Producing Disasters, Promoting Resilience (Stanford University Press, 2014)

57. H. Bergson H (Robin Durie, ed.), Duration and Simultaneity (Clinamen Press, Manchester, 1999). Originally published in French: Paris: Presses Universitaires de France, 1968

58. R. Descartes, D. Weissman, Discourse on the Method: And, Meditations on First Philosophy (Yale University Press, 1996)

59. G. Simondon, Du mode d'existence des objets techniques. Aubier (2012)

60. P. Virilio, La vitesse de la libération. Galilée (1995)

61. M. Onoda, H. Takahashi, Fukushima No. 2. Scrambled to avoid same fate as sister site Fukushima No 1, in Japan Times, September 102014

62. H. Takahashi, Y. Yukiko Maeda, Y. Shinohara, Yoshida's call on seawater kept reactor cool as Tokyo dithered, in Japan Times, September 142014

63. D. Lochbaum, E. Lyman, S.Q. Stranahan, Fukushima: The Story of a Nuclear Disaster (The New Press, 2014)

64. Y. Funabashi, K. Kitazawa, Fukushima in review: A complex disaster, a disastrous response. Bull. Atom. Sci. 68(2) (2012)

65. C. Perrow, The Next Catastrophe: Reducing Our Vulnerabilities to Natural, Industrial, and Terrorist Disasters (Princeton University Press, 2007)

66. R.J. Samuels, 3.11 Disaster and Change in Japan (Cornell University Press, 2013)

67. M. Edelstein, A. Wandersman, Contaminated Communities: The Social and Psychological Impacts of Residential Toxic Exposure (Westview Press, Boulder, CO, 1988)

68. F.H. Norris, S.P. Stevens, B. Pfefferbaum, K.F. Wyche, R.L. Pfefferbaum, Community Resilience as a Metaphor, Theory, Set of Capacities, and Strategy for Disaster Readiness. Am. J. Community Psychol. 41(1-2), 127-150 (2008) 
69. D. Woods, Creating Foresight: How Resilience Engineering Can Transform NASA's Approach to Risky Decision Making. Testimony on the Future of NASA for Committee on Commerce, Sciences and Transportation, John McCain Chair (2003)

70. A. Winston, Resilience in a hotter world. Harv. Bus. Rev. (2014)

71. H. Smet, P. Lagadec, J. Leysen, Disasters out of the box: a new ballgame? J. Conting. Crisis Manage. 20(3), 138-148 (2012)

72. ASIS, Organizational Resilience: Security, Preparedness, and Continuity Management Systems-Requirements with Guidance for Use (American Nationals Standards Institute, 2009)

73. D. Snowden, Complex acts of knowing - paradox, and descriptive self-awareness. Journal of Knowledge Management 6(2), 100-111 (2002)

74. J. Rasmussen, Risk management in a dynamic society: A modeling problem. Saf. Sci. 27, 183-213 (1997)

75. S. Dekker, E. Hollnagel, D. Woods, R. Cook, Resilience Engineering: New directions for measuring and maintaining safety in complex systems. Final Report, November 2008. Lund University School of Aviation (2008)

76. R. Westrum, Cultures with requisite imagination, in Verification and Validation of Complex Systems: Human Factors Issues, NATO ASI Series, ed. by J.A. Wise, V.D. Hopkin, P Stager, vol. 110 (Springer, Berlin, 1993), pp. 401-446

77. M.H. Schafer, T.P. Shippee, K.F. Ferraro, When does disadvantage not accumulate? Toward a sociological conceptualization of resilience. Schweizerische Zeitschrift für Soziologie 35(2), 231-251 (2009)

78. S.E. Hobfoll, Stress, Culture, and Community: The Psychology and Philosophy of Stress (Plenum, New York, 1998)

79. E.H. Powley, Reclaiming resilience and safety: Resilience activation in the critical period of crisis. Human Rel. 62(9) (2009)

80. K.M. Suttcliffe, T.J. Vogus, Organizing for resilience, in Positive Organizational Scholarship: Foundations of a New Discipline, ed. by K.S. Cameron, J.E. Dutton, R.E. Quinn (Berrett-Koehler, San Francisco, CA, 2003), pp. 94-110

81. F. Guarnieri, S. Travadel, Engineering thinking in emergency situations: a new nuclear safety concept. The Bulletin of the Atomic Scientists 70(6), 79 (2014)

Open Access This chapter is licensed under the terms of the Creative Commons Attribution 4.0 International License (http://creativecommons.org/licenses/by/4.0/), which permits use, sharing, adaptation, distribution and reproduction in any medium or format, as long as you give appropriate credit to the original author(s) and the source, provide a link to the Creative Commons license and indicate if changes were made.

The images or other third party material in this chapter are included in the chapter's Creative Commons license, unless indicated otherwise in a credit line to the material. If material is not included in the chapter's Creative Commons license and your intended use is not permitted by statutory regulation or exceeds the permitted use, you will need to obtain permission directly from the copyright holder.

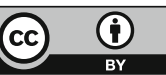

